\title{
NANOPARTICLE-ENABLED WIRELESS MONITORING AND CHARACTERIZATION OF DEGRADATION KINETICS IN PHARMACEUTICAL GELATIN FILMS
}

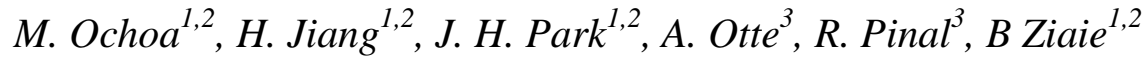 \\ ${ }^{1}$ Birck Nanotechnology Center, Purdue University \\ ${ }^{2}$ School of Electrical and Computer Engineering, Purdue University \\ ${ }^{3}$ College of Pharmacy, Purdue University
}

\begin{abstract}
Degradation kinetics of pharmaceutical excipient films effect their overall performance and drug release profile. Characterizing them is traditionally labor-intensive and time-consuming, requiring spectroscopy or periodic mass measurements. Here we present an alternative rapid technique for electrically (and wirelessly) measuring the polymeric matrix swelling and material degradation in aqueous media for characterizing functional films. The film is loaded with ferromagnetic nanoparticles and used as the core of a planar coil whose resonant frequency is monitored remotely. When placed in an aqueous solution, swelling and dissolution of the film induce contrasting changes in the capacitance and inductance of the coil, respectively, allowing identification of the swelling and dissolution phases. The dissolution profile of iron oxide-loaded gelatin is compared with spectrophotometry data, further demonstrating the technique can distinguish among films with various levels of crosslinking (showing a resonant frequency difference of $116 \mathrm{kHz}$ between completely noncrosslinked and fully crosslinked gelatin). The key characteristics of the film degradation kinetics can be captured within 20-30 minutes of data collection.
\end{abstract}

KEYWORDS: Wireless monitoring; rapid screening; pharmaceutical films, gelatin, ferrogelatin, degradation. 


\section{INTRODUCTION}

Tunable and environmentally-responsive pharmaceutical films offer a convenient vehicle for delivering drugs at specific locations in the body in a controlled fashion due to their biocompatibility, biodegradability, and modifiable properties [1-6]. As a result, researchers have developed a plethora of drug delivery systems, from ingestible capsules/tablets to topical and buccal delivery strips, which may rely on layered arrangements or coatings of such films to program the delivery of various drugs. For these systems, the dissolution and erosion/degradation kinetics of the excipient play a crucial role in the drug release profile from polymeric-matrix systems. Typically, these systems are characterized by spectroscopic absorbance of a released drug [7-12]. More importantly, the drug release can actually be thought of as a secondary process, whereby the drug solute migrates towards the polymer's outer surface and then to the release media. It is generally believed that the solute diffusion, polymeric matrix swelling, and material degradation kinetics are the main driving forces for solute diffusion from polymeric films/matrices [13]. Therefore, it would be advantageous to have a means to characterize the polymeric matrix swelling and degradation kinetics, rather than measuring the secondary process (solute diffusion and/or dissolution).

In one approach to characterize the swelling, the excipient film is removed from its solution and characterized at various time points during its dissolution by drying it and measuring its mass [8] and/or its thickness (by ellipsometry) [14,15]. Others have used a penetrometer or a video-microscope equipped with an image analyzer to visually distinguish between gelled and glassy regions in the film. While these techniques are suitable in many cases (e.g., small-scale quality control studies of film dissolution or drug release), there are several limitations rendering these methods suboptimal for high-throughput film screening; these include reliance on manual methods (and the resulting low sampling frequency), the need for complex fluidic setups (especially if attempting parallel experiments), and the 
requirement for continuous stirring (which may further alter the dissolution kinetics of the film).

A more convenient approach would be a non-contact (i.e., non-invasive), automated, electrical method for assessing film integrity of multiple samples during dissolution. Electrical techniques are advantageous due to their ease of interfacing with a variety of data acquisition systems commonly found in many laboratories. Unfortunately, most pharmaceutical excipients do not possess strong electromagnetic properties that can be monitored. To circumvent this shortcoming, we have developed a sensing scheme for wirelessly monitoring the matrix swelling and degradation kinetics of established pharmaceutical film materials by incorporating biocompatible $[16,17]$ ferromagnetic nanoparticles; as a result, the films exhibit a measurable degradation-dependent change in the resonant frequency of the coil/film device. In this paper, we present a proof-of-concept demonstration of this technique. Here, we use gelatin (of various degrees of crosslinking: un-, partially-, fully-crosslinked gelatins [18]) loaded with iron oxide nanoparticles to create "ferrogelatin". When the film is placed in close proximity to a resonant (LC) circuit, the swelling and dissolution of the ferrogelatin can be remotely monitored by measuring the resonant frequency of the circuit [19]. This method offers additional advantages over other techniques like those based on detecting and differentiating between the initial swelling and the subsequent dissolution phase, including a higher temporal resolution, and spatial scalability. Rather than inspecting the film manually (which is typically done at intervals of 5-15 minutes), it is possible to conduct electrical measurements with a sampling rate on the order of seconds or faster, thus allowing data collection in time intervals shorter than one minute. Furthermore, by virtue of being completely electrical, this technique is suitable for implementing as a batch-screening arrangement (e.g., with a multiplexing circuit) to conduct a simultaneous screening of multiple gelatin samples, as depicted in Figure 1. 


\section{OPERATION PRINCIPLE}

The working principle of the sensing scheme is illustrated in Figure 2. Monitoring is achieved by placing a flat coil adjacent to the ferrogelatin film as it dissolves in the dissolution medium (e.g., $37^{\circ} \mathrm{C}$ water or saline). Thus, the ferrogelatin behaves as the core of the coil, increasing its inductance. Once placed in an aqueous medium, the ferrogelatin film initially absorbs water, thus also altering the local permittivity (2-4 for dry proteins, but about 80 for water) [20] around the inductor. Similar to other soluble films when exposed to aqueous environments, physical degradation of the gelatin film proceeds by two driving mechanisms: swelling and subsequent dissolution. As a result, when the physical properties of the film change (i.e., by swelling or erosion), the local magnetic permeability and permittivity change. Such changes alter in turn the inductance or capacitance of the LC tank. These changes are be detected wirelessly by monitoring the self-resonant frequency of the inductor via a second (read-out) coil using the phase-dip method [19].

A unique feature of this monitoring technique is the integration of inductive as well as capacitive sensing, which in combination can be used to differentiate among the various stages of film degradation, i.e., initial swelling when placed in the medium, transition to dissolution, and complete dissolution. This type of differentiation is possible since the swelling and degradation of ferrogelatin cause the resonant frequency of the system to change in opposing directions. To understand these effects, we consider the ferrogelatin and coil system as an LC circuit ( $\mathrm{L}$ being the inductance of the coil and $\mathrm{C}$ in this case representing the parasitic capacitances associated with it, i.e., the capacitors between various turns with electric flux going through the ferrogelatin film or surrounding aqueous media), whose self-

resonant frequency is given by $f_{0}=\frac{1}{\sqrt{L C}}$. The inductance depends primarily on the geometry of the coil and the amount of ferromagnetic material (i.e., permeability) in the film, whereas 
fringe/parasitic capacitance is a function of the geometric design of the coil and the permittivity of the ferrogelatin film (which as mentioned above depends mainly on its water content). When ferrogelatin is immersed in an aqueous medium the capacitance and inductance are affected at different stages: permittivity, and hence capacitance, is primarily affected during the initial absorption of water following immersion, whereas inductance primarily during the degradation phase. As shown in Figure 2a, swelling of ferrogelatin increases fringe capacitance in the sensing coil due to the elevated relative permittivity (i.e., of water), resulting in a reduced resonant frequency. In contrast, as gelatin degrades, the loss of ferro-particles reduces the magnetic permeability (and hence inductance), resulting in an increased frequency, Figure $2 \mathrm{~b}$. Thus, by observing the change in the resonant frequency, it is possible to identify the various phases of the film degradation process.

The resonant frequency can be detected through inductive coupling that the external coil energizes this LC circuit, which provides a load impedance that is reflected back to the external coil [19]. Briefly, the impedance phase dip, $\Delta \varphi_{D I P} \cong \arctan \left(\frac{2 \pi f_{0} M^{2}}{L_{e} R_{S}}\right)$, is a function of the resonance frequency $f_{0}$, the mutual inductance between the external coil and LC circuit, $\mathrm{M}$, the inductance of the external coil $L_{e}$, and series resistance of the LC circuit, $R_{S}$. At the resonant frequency of the LC circuit, the impedance phase dip is maximized when $R_{S}$ is minimized; thus, by monitoring the overall phase change of the external coil due to the reflected impedance, it is possible to detect the resonance frequency of the LC circuit.

\section{EXPERIMENTAL}

\section{$3.1 \quad$ Test platform fabrication}

Ferrogelatin films can be characterized by creating a simple setup via the fabrication process described in Figure 3. The system uses a polyimide-coated planar coil (double layer, 
Parlex Corporation) as the primary coil. These are high-Q coils allowing for easy wireless detection and high precisions frequency readout; however, their polyimide coating prevents adhesion to other materials, including ferrogelatin. Thus, as a first step, the coil is embedded in a substrate to which the ferrogelatin can adhere (e.g., silicone). For this we cast $21.5 \mathrm{mg}$ of PDMS (polydimethylsiloxane, Dow Corning Sylgard 184) pre-polymer in a laser-machined acrylic well (20 mm diameter) and partially cure it on a hot-plate $\left(70{ }^{\circ} \mathrm{C}, 30 \mathrm{~min}\right)$ to create a $150 \mu$ m-thick PDMS layer at the bottom (Figure 3a). Next, the coil is placed on the PDMS (Figure 3b). Due to partial crosslinking of the PDMS, the polyimide-coated coil adheres to the PDMS surface. The coil is then encapsulated with an additional volume of PDMS (Figure 3c). The PDMS is then allowed to crosslink completely in an oven $\left(80^{\circ} \mathrm{C}, 2 \mathrm{~h}\right)$. Next, the PDMS is surface-treated by oxygen plasma, and a water-impermeable film (i.e., PET, PP2500, 3M). This PET film, which serves the role of sample holder for the film, is partially bonded (but removable) to the PDMS surface (Figure 3d). The resulting structure is a reusable test fixture upon which ferrogelatin films can be attached (i.e, by bonding them to the PET using a thin adhesive layer, e.g., Loctite 3105) for degradation analysis of the films (Figure 2e-f).

\subsection{Preparation of ferrogelatin}

Ferrogelatin films are fabricated using gelatin (Type A from porcine skin, 300 bloom, Sigma Aldrich) and iron oxide nanoparticles (<50 nm diameter, Sigma Aldrich). A $30 \%$ w/v aqueous solution of gelatin is prepared at $50-55^{\circ} \mathrm{C}$. The iron oxide nanoparticles are then added in a $10 \% \mathrm{w} / \mathrm{w}$ ratio to gelatin. To disperse the nanoparticles the solution is homogenized for (using a $50 \%$ duty cycle with a period of one minute, i.e., 30 seconds ON/30 seconds OFF) for a total of 5 minutes. Next, $10 \mathrm{~mL}$ of the solution is cast into a petri dish and allowed to form a film during 24 hours; this results in non-crosslinked ferrogelatin films. To create ferrogelatin with various dissolutions rates, similar ferrogelatin solutions are 
cast and placed in a vacuum chamber along with $10 \mathrm{~mL}$ of formaldehyde. The ferrogelatin is then allowed to crosslink in a formaldehyde vapor environment for a period of 30 minutes (for partially-crosslinked ferrogelatin) or for $12 \mathrm{~h}$ (for fully-crosslinked ferrogelatin). These films are subsequently dried at room temperature for $24 \mathrm{~h}$. Once cured, ferrogelatin films are laser-machined into discs (20 mm diameter) and each disc is attached to a test platform (on the PET side, closest to the coil) (Figure 3e-f). The fabricated sensor with the test fixture is $20 \mathrm{~mm}$ in diameter and $2.5 \mathrm{~mm}$ in thickness.

\subsection{Film degradation experiments}

Ferrogelatin degradation occurs in warm aqueous solutions, typically in the range of $35-40{ }^{\circ} \mathrm{C}$ for this type of gelatin [21]. To mimic physiological conditions in humans, water was heated to $37^{\circ} \mathrm{C}$; however, the specific temperature used should be set based on the target body temperature of the target animal species. Each ferrogelatin film (mounted on a coil platform) was tested as follows. The platform was immersed in water at $37{ }^{\circ} \mathrm{C}$ but maintained at close proximity $(2 \mathrm{~mm})$ to the secondary readout coil connected to an impedance analyzer (Agilent Technologies, 4396B). As the ferrogelatin swelled and dissolved, the resonant frequency of the PDMS-embedded coil was measured wirelessly by the phase-dip method [19]. The change in resonant frequency was recorded over time (up to 6 hours) as the film degraded. Photographs of the degradation process were also obtained to visually follow degradation. The experiments were conducted at a $\mathrm{pH}$ of 7.4 as a proof of concept demonstration under simulated physiological conditions; however, the technique can be applied to other physiologically-relevant $\mathrm{pH}$ values to evaluate the film dissolution in specific target environments.

For comparison, the degradation of the film was also measured by spectrophotometry (UV-260, Shimadzu). Solution samples from degrading films (noncrosslinked) were taken at various time intervals, and their UV absorbance was recorded at 
$285 \mathrm{~nm}$.

\section{RESULTS AND DISCUSSION}

A photograph of a completed ferrogelatin-loaded test platform prior to testing is shown in Figure 4. The bond between the PET and the PDMS is sufficiently strong to prevent unintentional detachment during the dissolution experiments (verified by Scotch tape peel test and subsequently by the dissolution experiments), but can be removed to reuse the PDMS-coil structure. Figure 5 shows time-lapse photographs of one such (partiallycrosslinked) ferrogelatin film at various stages of degradation in $37^{\circ} \mathrm{C}$ water. During the first few minutes, the ferrogelatin maintains its shape (Figure 5a); however, due to its hygroscopic character, the ferrogelatin absorbs water prior to the start of the main dissolution phase. After 52 minutes, the ferrogelatin is clearly in the dissolution-dominated phase and remains in this phase until all of the ferrogelatin is dissolved (Figure 5b-f). For completely crosslinked and non-crosslinked ferrogelatin, the duration of each phase can be expected to be longest and shortest, respectively.

Quantitative measurements using resonant frequency also reflect the various stages of degradation. Figure 6 shows the change in resonant frequency of a partially-crosslinked ferrogelatin film over six hours (average of three samples, with shades showing 1 SD errors). The magnitudes of the changes in frequency are sufficiently large to be easily detectable using a laboratory impedance analyzer. The resonant frequency drops initially upon immersion in water, but it gradually rises over time as the film dissolves. The inset highlights the behavior during the first five minutes, showing a total decrease in resonant frequency of $259 \mathrm{kHz}$. Since the ferrogelatin is primarily absorbing water during this stage (limited dissolution) the drop in resonant frequency can be attributed to increased fringe capacitance between the turns of the coil, arising from an increase in relative permittivity within the 
ferrogelatin as it saturates with water [20]. Following a brief transition phase, the remainder of the testing time exhibits a monotonically increasing resonant frequency; the total increase during this time is $155 \mathrm{kHz}$. At this stage, the permittivity has already saturated. Therefore, the increase in resonant frequency can be attributed solely to the inductive effect, i.e., increasing frequency due to the dissolution of ferrogelatin (reduced magnetic permeability). It is interesting to note that the steady-state resonant frequency is $104 \mathrm{kHz}$ lower than the initial value. This can be understood as simply environment-dependent differences in fringe capacitance, since the initial value represents the ferrogelatin film surrounded by air (both with $\varepsilon_{r} \approx 1-4$ ), whereas for steady-state during the test, the film is saturated with water or has been completely eroded and replaced by water (both cases with permittivity close to water, $\varepsilon_{r} \approx 80$ ).

To verify the degradation kinetics of the ferrogelatin samples, we conducted spectroscopic absorbance measurements during degradation and compared them to electromagnetic (frequency) measurements. Figure 7 shows the averaged results using three non-crosslinked ferrogelatin films for each measurement technique. The UV absorbance profile exhibits a logarithmic trend, a typical drug release profile among spectroscopic studies of pharmaceutical films. Meanwhile, the frequency data show the same trend seen in Figure 6, i.e., a steep drop in frequency for 15 minutes (as the gelatin absorbs water), followed by a gradual increase in frequency afterward (as the film material is eroded/dissolved).

Additional experiments with ferrogelatin samples of various degrees of crosslinking further elucidate the ability to characterize ferrogelatin films via this wireless electrical method. Figure 8 shows the change in resonant frequency for samples of non-crosslinked, partially crosslinked, and fully crosslinked ferrogelatin (measurements for each type were conducted in triplicates). One feature of the data demonstrates the effect of varying the degree of crosslinking: the maximum frequency drop during the initial swelling phase $(-151 \mathrm{kHz}$ for 
non-crosslinked, $-258 \mathrm{kHz}$ for partially crosslinked, and $-267 \mathrm{kHz}$ for fully crosslinked ferrogelatin). This is an important aspect, since the release mechanism in pharmaceutical films is known to critically depend on the transition from swelling to degradation/diffusion. Current methods for pinpointing the transition actually rely on bracketing it by testing different samples at discrete time intervals. Accordingly, material characterization involves permanently removing a film sample from the medium in order to perform the particular characterization test. Alternatively (if the particular characterization test allows it) the film sample is withdrawn from the system, tested and returned to the system. Such disturbance may affect the swelling/degradation properties. With our method, it is possible to identify the transition wirelessly and continuously during swelling/degradation (as drug release occurs) for each film sample. The magnitude of the drop increases with the level of crosslinking, with a difference of $116 \mathrm{kHz}$ between the two extremes (non-crosslinked vs. completely crosslinked). This behavior is expected since increased crosslinking density decreases the diffusion coefficient of water in films such as gelatin [22], which delays the onset of the dissolution phase. In contrast, in the non-crosslinked films, the dissolution phase can be expected to overtake swelling at an earlier time, limiting the maximum frequency drop. The differences in frequency drops result in a clear separation between the curves during the dissolution phase (positive slope region). As a result, it is also possible to accurately differentiate among various levels of crosslinking at any time between the minimum frequency and the saturation time of the curve, i.e., the level of crosslinking induces a signature for ferrogelatin films that is electrically-measurable in a timely manner. For these experiments, 20-30 minutes of data collection is appears sufficient for assessing the degree of crosslinking as well as the two phases of degradation. 


\section{CONCLUSIONS}

A non-contact electromagnetic technique for monitoring material degradation in pharmaceutical films has been developed, whereby a ferromagnetic nanoparticle loaded film is bonded to a planar coil while its self-resonant frequency is wirelessly measured to delineate the initial swelling and subsequent dissolution/degradation phases of the film in an aqueous environment. In comparison to the traditional spectrophotometric methods used for measuring drug release, the electrical nature of this scheme enables its adaptation for highthroughput screening of films, providing precise information about the matrix swelling and degradation kinetics of the film. Specifically, the approach presented here provides unequivocal detection of the swelling-diffusion transition (a critical factor of the release mechanism) by finding a local minimum in the frequency curve. All without disturbing the sample or removing the dissolution medium. In contrast, traditional spectrophotometric methods do not offer such an unambiguous criterion for locating the transition point. Iron oxide-loaded gelatin (ferrogelatin) was used a proof-of-concept demonstration (the technique can also be applied to other pharmaceutical materials). Experiments revealed that a 20-30 minute data collection period is sufficient to record the key features of the film degradation. This technique has the potential for becoming a standard pharmaceutical film characterization method with significantly improved temporal efficiency as compared to current standards (e.g., Flory-Rehner swelling, which can take days [23-25]). Furthermore, it may be possible to reduce the response time by micro-patterning the gelatin film (as our group has shown with other hydrogel materials [26]), but this will require further characterization to develop a model/correlation between micro-patterns and standard (un-patterned) pharmaceutical film. 


\section{ACKNOWLDGEMENTS}

The authors thank to the staff of Birck Nanotechnology Center and the Dane O. Kildsig Center for Pharmaceutical Processing Research (CPPR) at Purdue University for their technical support. Funding for this work was provided in part by the National Science Foundation under grant EFRI-BioFlex \#1240443 and by the 2013 Discovery Park Faculty Research Fellowship Grant awarded to Prof. Pinal and Prof. Ziaie. 


\section{REFERENCES}

[1] K. Sato, K. Yoshida, S. Takahashi, J. Anzai, pH- and sugar-sensitive layer-by-layer films and microcapsules for drug delivery., Adv. Drug Deliv. Rev. 63 (2011) 809-21.

[2] S.A. Sukhishvili, Responsive polymer films and capsules via layer-by-layer assembly, Curr. Opin. Colloid Interface Sci. 10 (2005) 37-44.

[3] Y. Ikada, Y. Tabata, Protein release from gelatin matrices., Adv. Drug Deliv. Rev. 31 (1998) 287-301.

[4] N. Kavanagh, O.I. Corrigan, Swelling and erosion properties of hydroxypropylmethylcellulose (Hypromellose) matrices--influence of agitation rate and dissolution medium composition., Int. J. Pharm. 279 (2004) 141-52.

[5] Z. Ruszczak, Collagen as a carrier for on-site delivery of antibacterial drugs, Adv. Drug Deliv. Rev. 55 (2003) 1679-1698.

[6] A. Domb, S. Amselem, J. Shah, M. Maniar, Degradable polymers for site-specific drug delivery, Polym. Adv. Technol. 3 (1992) 279-292.

[7] J. Siepmann, H. Kranz, R. Bodmeier, N.A. Peppas, HPMC-matrices for controlled drug delivery: a new model combining diffusion, swelling, and dissolution mechanisms and predicting the release kinetics., Pharm. Res. 16 (1999) 1748-56.

[8] T.D. Reynolds, S.H. Gehrke, A.S. Hussain, L.S. Shenouda, Polymer erosion and drug release characterization of hydroxypropyl hethylcellulose matrices, J. Pharm. Sci. 87 (1998) 1115-1123.

[9] H. Kim, R. Fassihi, Application of binary polymer system in drug release rate modulation. 2. Influence of formulation variables and hydrodynamic conditions on release kinetics., J. Pharm. Sci. 86 (1997) 323-8.

[10] B. Narasimhan, N. a Peppas, Molecular analysis of drug delivery systems controlled by dissolution of the polymer carrier., J. Pharm. Sci. 86 (1997) 297-304.

[11] J. Luypaert, D.L. Massart, Y. Vander Heyden, Near-infrared spectroscopy applications in pharmaceutical analysis., Talanta. 72 (2007) 865-83.

[12] S.G. Kazarian, J. van der Weerd, Simultaneous FTIR spectroscopic imaging and visible photography to monitor tablet dissolution and drug release., Pharm. Res. 25 (2008) 853-60.

[13] D.Y. Arifin, L.Y. Lee, C.-H. Wang, Mathematical modeling and simulation of drug release from microspheres: Implications to drug delivery systems, Adv. Drug Deliv. Rev. 58 (2006) 1274-1325.

[14] E. Vázquez, D.M. Dewitt, P.T. Hammond, D.M. Lynn, Construction of Hydrolytically-Degradable Thin Films via Layer-by-Layer Deposition of Degradable Polyelectrolytes, J. Am. Chem. Soc. 124 (2002) 13992-13993.

[15] K.C. Wood, J.Q. Boedicker, D.M. Lynn, P.T. Hammond, Tunable Drug Release from Hydrolytically Degradable Layer-by-Layer Thin Films, (2005) 1603-1609.

[16] L.H. Reddy, J.L. Arias, J. Nicolas, P. Couvreur, Magnetic Nanoparticles: Design and Characterization, Toxicity and Biocompatibility, Pharmaceutical and Biomedical 
Applications, Chem. Rev. 112 (2012) 5818-5878.

[17] T.K. Jain, M.K. Reddy, M.A. Morales, D.L. Leslie-Pelecky, V. Labhasetwar, Biodistribution, Clearance, and Biocompatibility of Iron Oxide Magnetic Nanoparticles in Rats, Mol. Pharm. 5 (2008) 316-327.

[18] A. Duconseille, T. Astruc, N. Quintana, F. Meersman, V. Sante-Lhoutellier, Gelatin structure and composition linked to hard capsule dissolution: A review, Food Hydrocoll. 43 (2015) 360-376.

[19] O. Akar, T. Akin, K. Najafi, A wireless batch sealed absolute capacitive pressure sensor, Sensors Actuators A Phys. 95 (2001) 29-38.

[20] S.C. Harvey, Treatment of electrostatic effects in macromolecular modeling., Proteins. 5 (1989) 78-92.

[21] Y. Tabata, Y. Ikada, Vascularization effect of basic fibroblast growth factor released from gelatin hydrogels with different biodegradabilities, Biomaterials. 20 (1999) 2169-2175.

[22] R.A. De Carvalho, C.R.F. Grosso, Characterization of gelatin based films modified with transglutaminase, glyoxal and formaldehyde, Food Hydrocoll. 18 (2004) 717-726.

[23] M. Barlkani, C. Hepburn, Determination of crosslink density by swelling in the castable polyurethane elastomer based on 1/4-cyclohexane diisocyanate and paraphenylene, Iran. J. Polym. Sci. Tech. 1 (1992) 1-5.

[24] C.M. Ofner, W.A. Bubnis, Chemical and swelling evaluations of amino group crosslinking in gelatin and modified gelatin matrices., Pharm. Res. 13 (1996) 1821-7.

[25] R. Huggins, Statistical Mechanics of Cross-Linked Polymer Networks, J. Adhes. 11 (1943) 521-526.

[26] S.H. Song, J.H. Park, G. Chitnis, R.A. Siegel, B. Ziaie, A wireless chemical sensor featuring iron oxide nanoparticle-embedded hydrogels, Sensors Actuators B Chem. 193 (2014) 925-930. 


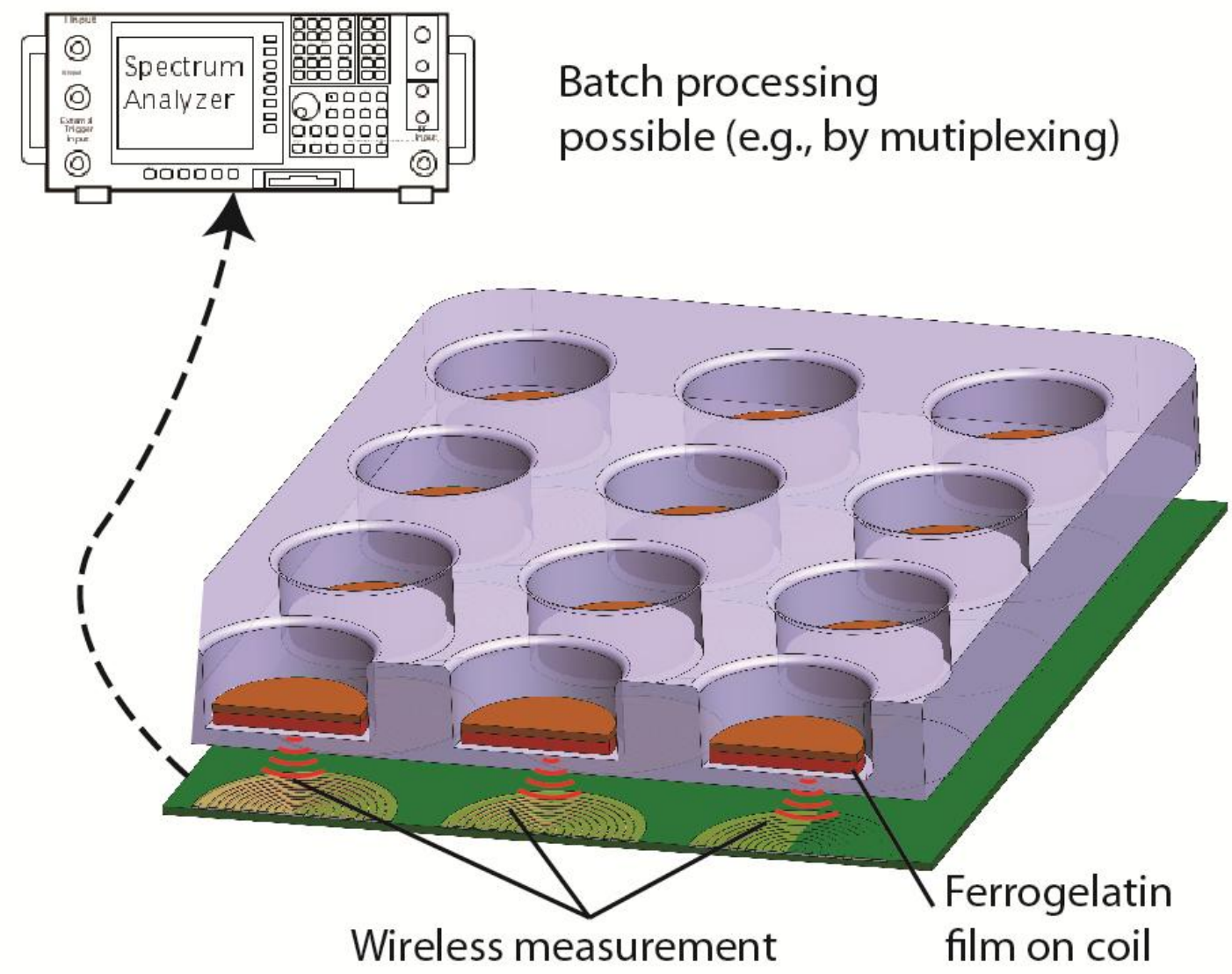

Figure 1. Schematic showing the use of the described measurement technique adapted for multi-sample, wireless, high-throughput screening of pharmaceutical films. Drug excipient films are loaded with ferromagnetic particles, cast into dry discs, and mounted on the electromagnetic test platforms. 
(a) Swelling: $\varepsilon_{\mathrm{r}} \uparrow C \uparrow \mathrm{f} \downarrow$

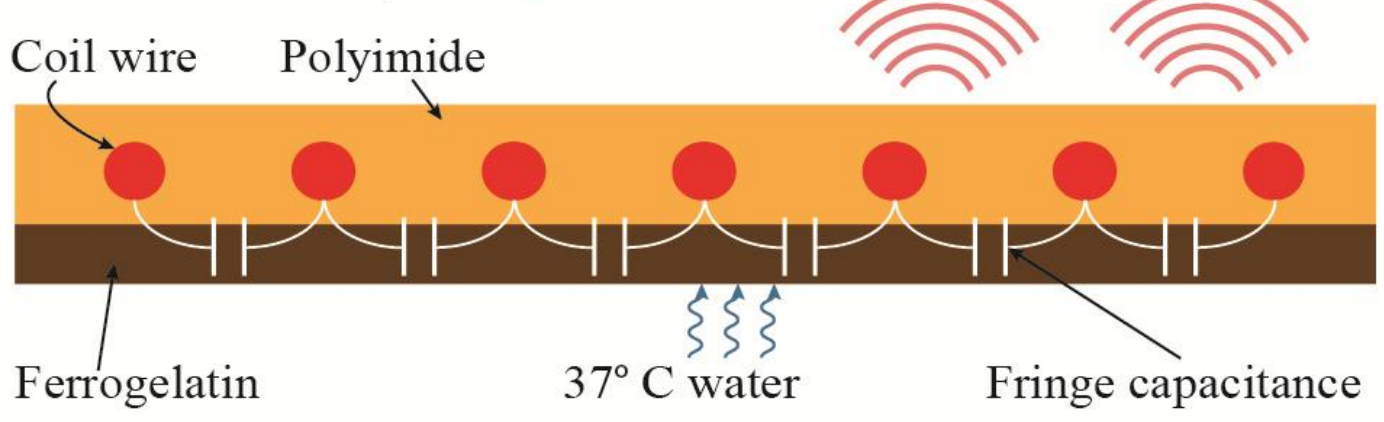

(b) Dissolving: $\mu_{\mathrm{r}} \downarrow \downarrow \downarrow \mathrm{f} \uparrow$

To readout coil

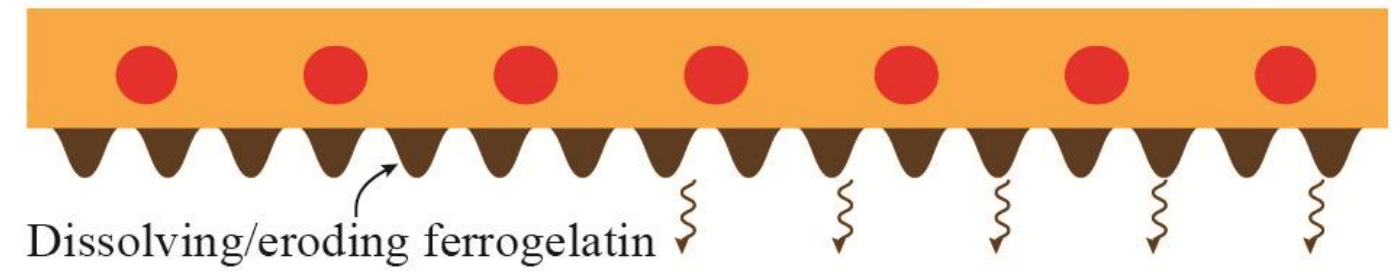

Figure 2. Illustration of the sensing mechanism used to characterize the ferrogelatin degradation with time. The diagram shows the cross-section of a polyimide-embedded coil with an attached ferrogelatin film; (a) initially ferrogelatin film swells upon immersion in water, changing the effective dielectric constant of the film (from about 2-4 for dry proteins to about 80 for water), causing an increase in the fringe capacitance of the coil (depicted as white capacitors) and a drop in its resonant frequency; (b) subsequent dissolution of the film erodes away the ferromagnetic material, decreasing the magnetic permeability of the coil and thus increasing its resonant frequency. 
(a)

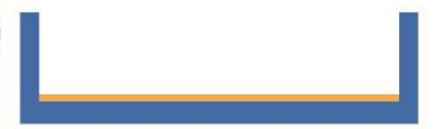

(b)

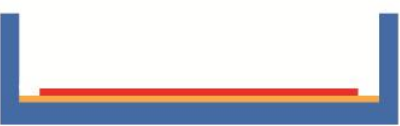

(c)

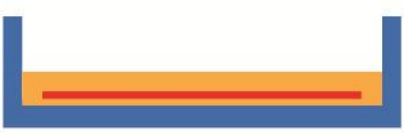

\section{PDMS Acrylic mold $\square$ PET}

$\square$ Ferrogelatin $\square$ Sensing coil (d)

(e)

(f)

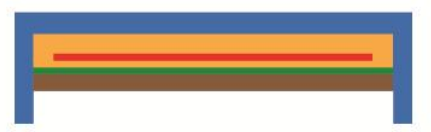

Figure 3. Fabrication process of the ferrogelatin testing platform. (a) Laser-machine an acrylic mold for creating the PDMS structure, and cast PDMS to form a $150 \mu \mathrm{m}$ layer. (b) Partially cure the PDMS layer and subsequently attach a polyimide-coated coil; (c) encapsulate the coil in more PDMS (cast and cure); (d) insulate with a partially plasmabonded PET sheet $(100 \mu \mathrm{m}$ thick) to prevent moisture penetration into the polyimide; (e) prepare a ferrogelatin disc by casting, curing, and laser-machining dry ferrogelatin, then attach it to the PET using a UV curable adhesive; (f) the structure is ready for test using a secondary read-out coil and an impedance analyzer. 


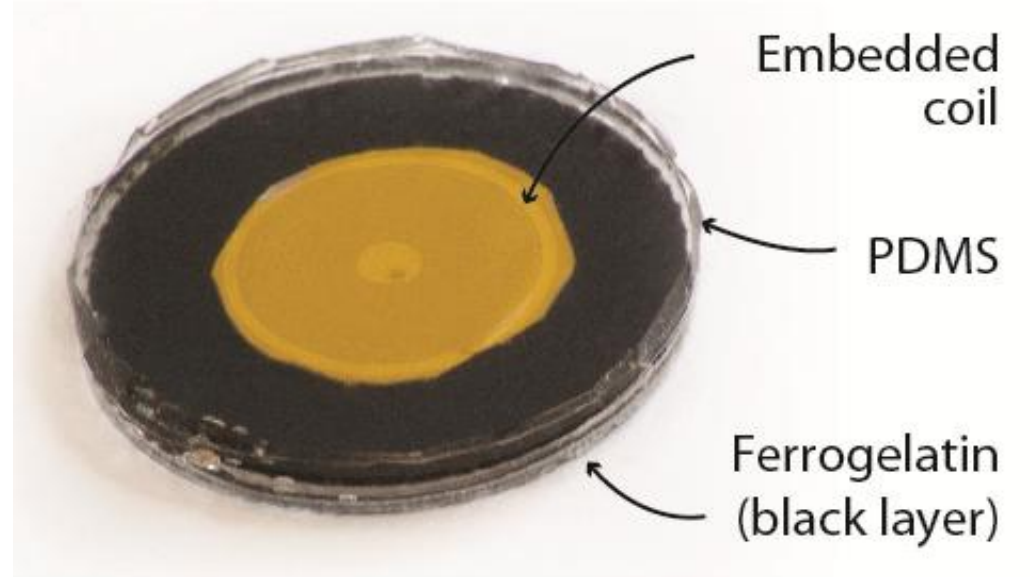

Figure 4. Test platform comprising a commercial polyimide-coated copper coil, which is encapsulated in PDMS and attached to a disc of ferrogelatin (lined with PET). The coilPDMS serves as a reusable "chip" for characterization of ferromagnetic films. 

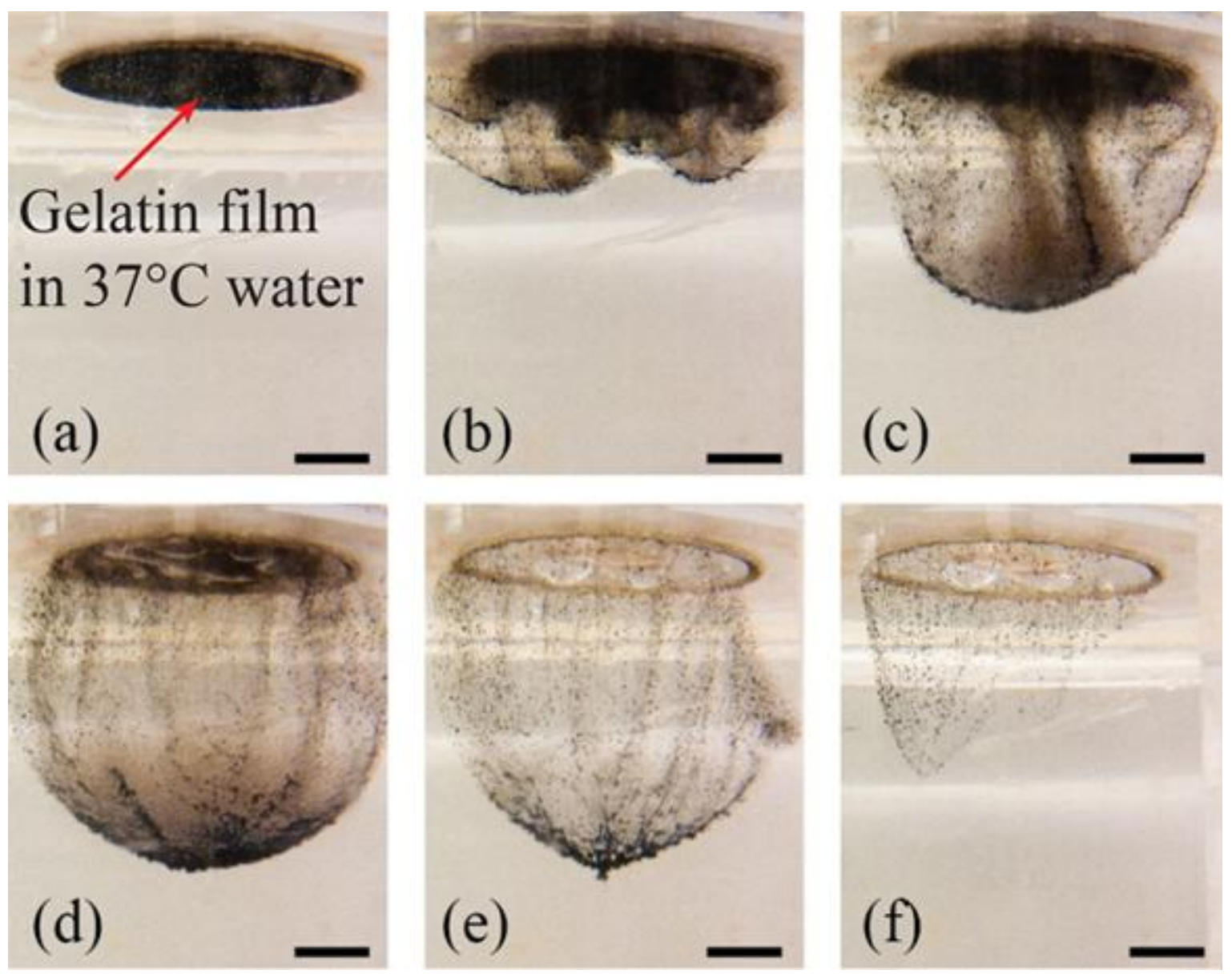

Figure 5. Photographs of partially-crosslinked ferrogelatin degradation in $37{ }^{\circ} \mathrm{C}$ water at various times (a) $0 \mathrm{~min}$; (b) $52 \mathrm{~min}$; (c) $60 \mathrm{~min}$; (d) $84 \mathrm{~min}$; (e) $220 \mathrm{~min}$; (f) $360 \mathrm{~min}$; scale bar: $5 \mathrm{~mm}$. Swelling occurs primarily during the first snapshot; dissolution occurs during the subsequent ones. The readout coil (not visible) is above the sample, within $2 \mathrm{~mm}$ from the PDMS testing platform. A different angle of (a) is available in the supplementary section, showing the read-out coil above the sample. 


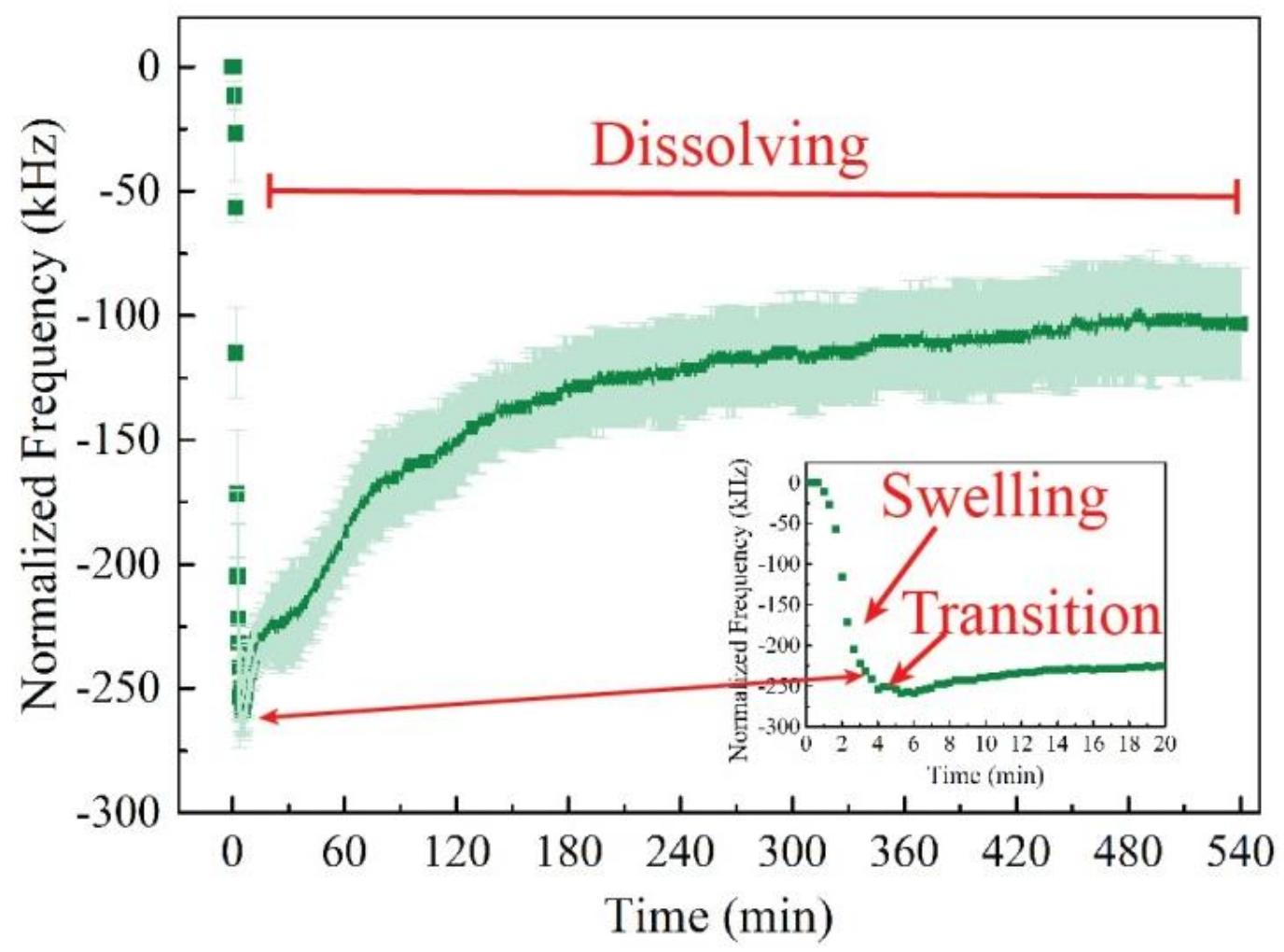

Figure 6. Temporal response of the sensing coil resonant frequency showing the various phases of ferrogelatin degradation; for these samples $(n=3)$, swelling (decreasing frequency) occurs during the first 5 minutes (see inset), whereas dissolution (increasing frequency) occurs for the remainder of the time following a brief transition phase. The shaded region represents $1 \mathrm{SD}$ errors for three samples. 


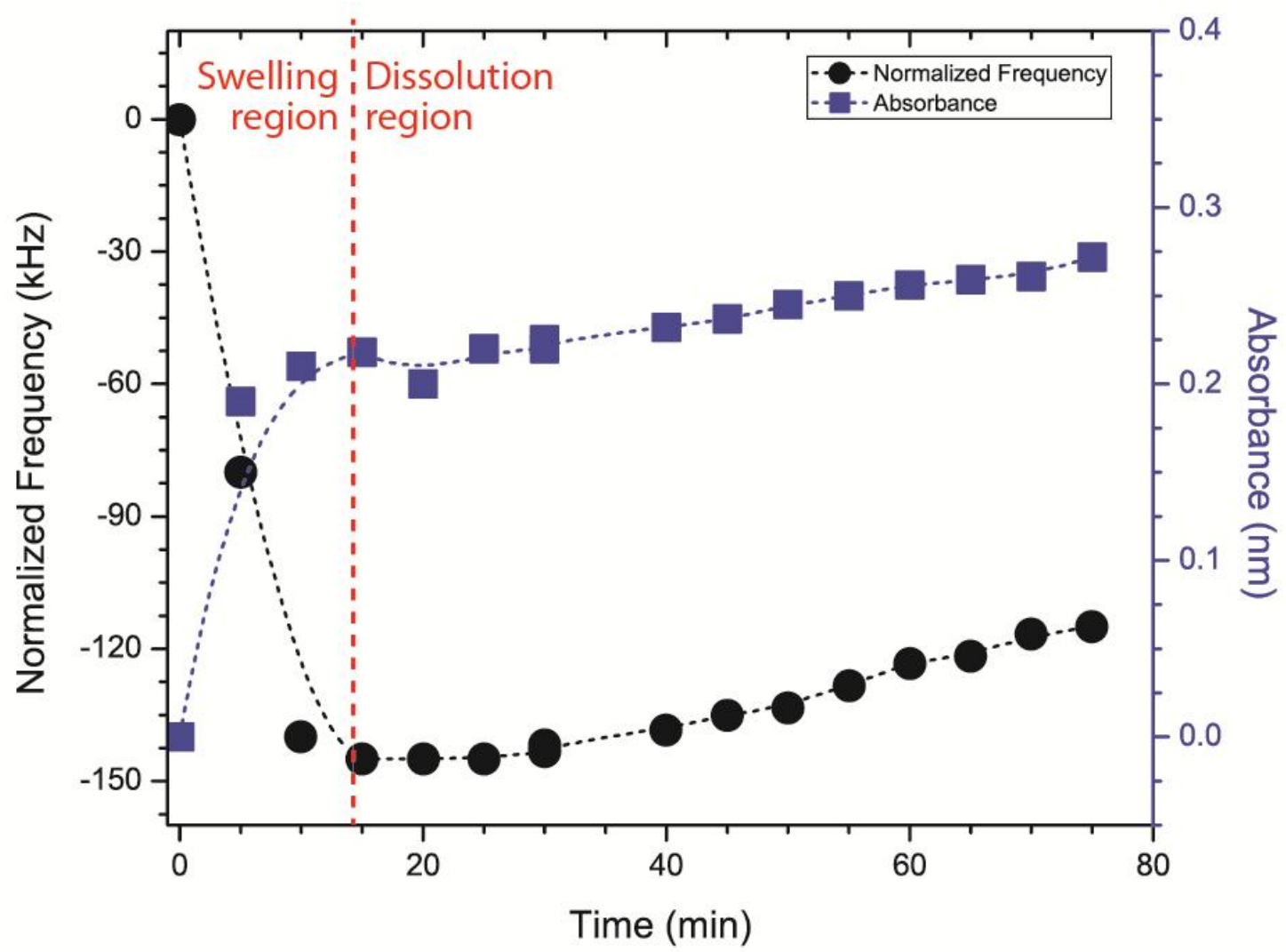

Figure 7. Spectroscopic data and change in the resonant frequency for a sample during 80 minutes of water immersion. The data confirm a correlation between spectroscopic techniques and the electrical one. Degradation is swelling-dominated for the first 15 minutes but dissolution-dominated afterwards. The frequency curve mirrors the absorbance (large initial change followed by a gradual monotonic rise) for the duration of the test. 


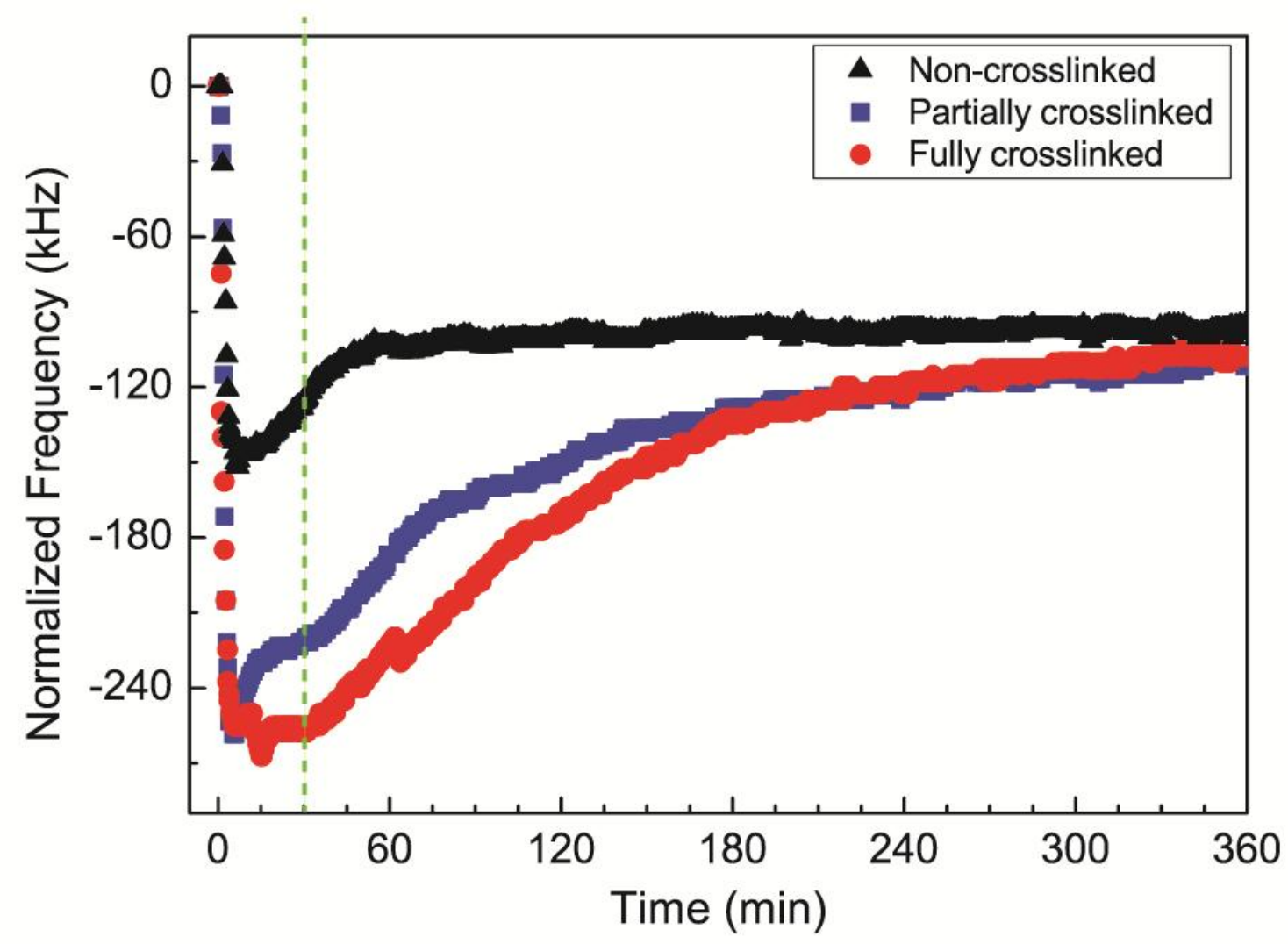

Figure 8. Comparison of the temporal response of ferrogelatin film swelling and degradation for three degrees of crosslinking. The data reveal differences in maximum frequency drop $(-151 \mathrm{kHz}$ for non-crosslinked, $-258 \mathrm{kHz}$ for partially crosslinked, and $-267 \mathrm{kHz}$ for fully crosslinked ferrogelatin), as well as saturation trend among the three samples. Thirty minutes (green dotted line) of data collection per sample is sufficient to accurately distinguish between non-crosslinked and fully-crosslinked ferrogelatin as well as intermediate levels of crosslinking, while simultaneously permitting identification of the swelling and dissolution phases of each. Data in this plot was collected in triplicate for each level of crosslinking. 


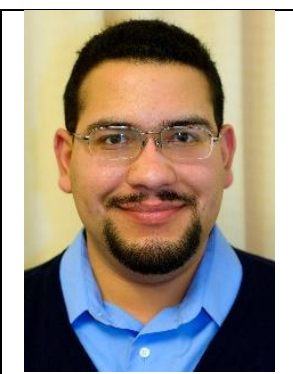

Manuel Ochoa received the B.S. degree in electrical engineering from the California Institute of Technology, Pasadena, CA in 2009 and the M.S. degree in electrical and computer engineering from Purdue University, West Lafayette, IN in 2012. He is currently pursuing a Ph.D. degree in electrical and computer engineering at Purdue University. Since 2009 he has been a research assistant with the Ziaie Biomedical Microdevices Laboratory at Purdue. His research focuses on the integration of common materials (e.g. paper, tape, yeast) for the development low-cost, multifunctional, microsystem platforms for biomedical applications, including transdermal drug delivery and dermal wound healing.

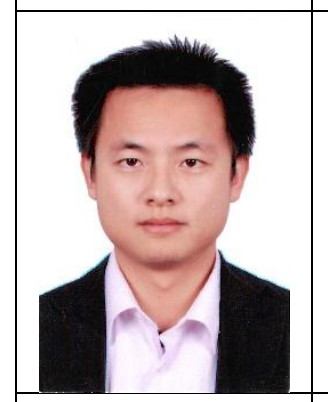

Hongjie Jiang received the M.S. degree in electrical engineering from the Beijing Institute of Technology, Beijing, China in 2005. He is currently pursuing a Ph.D. degree in electrical and computer engineering at Purdue University. Since 2013 he has been a research assistant with the Ziaie Biomedical Microdevices Laboratory at Purdue. His research focuses on the wireless sensing of degradation in polymeric film for drug delivery, low-cost and flexible microsystem platforms for biomedical application.

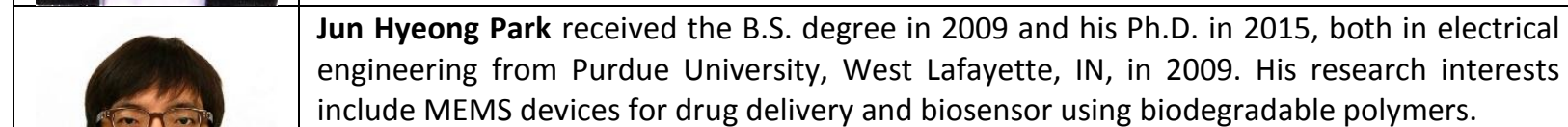
include MEMS devices for drug delivery and biosensor using biodegradable polymers.

Andrew Otte received the B.S. degree in Pharmaceutical Sciences in 2005 and a PhD in Pharmaceutics in 2011, both from Purdue University, West Lafayette, IN. He is currently working as a postdoctoral scientist in biomedical engineering at Purdue University. His research interests lie in the development of multi-layered/functional polymer matrix tablets, fabrication of micro-nanoparticles, and depot formulations for long-term drug delivery.

Rodolfo Pinal Rodolfo Pinal is Associate Professor in Industrial and Physical Pharmacy and Director of the Dane O. Kildsig Center for Pharmaceutical Processing Research at Purdue University. He has 13 years of experience in the pharmaceutical industry. His research covers solubility and dissolution phenomena with focus on the design of responsive composites for optimizing drug release and bioavailability for application in the development of patient-centered, precision pharmaceuticals.

$\triangle$

Babak Ziaie received his doctoral degree in Electrical Engineering from the University of Michigan in 1994. From 1995 to 1999 he was a postdoctoral-fellow and an assistant research scientist at the Center for Integrated Microsystems (CIMS) of the University of Michigan. He subsequently joined the Electrical and Computer Engineering Department of the University of Minnesota as an assistant professor (1999-2004). Since January 2005, he has been with the School of Electrical and Computer Engineering at Purdue University where he is currently a professor. His research interests are related to the biomedical applications of MEMS and Microsystems (BioMEMS). 